\title{
A personalized exercise trainer for the elderly
}

Gabriele Bleser, Daniel Steffen, Markus Weber, Gustaf Hendeby, Didier Stricker, Laetitia

Fradet, Frédéric Marin, Nathalie Ville and Francois Carré

\section{Linköping University Post Print}

\section{Tweet}

N.B.: When citing this work, cite the original article.

Original Publication:

Gabriele Bleser, Daniel Steffen, Markus Weber, Gustaf Hendeby, Didier Stricker, Laetitia Fradet, Frédéric Marin, Nathalie Ville and Francois Carré, A personalized exercise trainer for the elderly, 2013, Journal of Ambient Intelligence and Smart Environments, (5), 6, 547-562. http://dx.doi.org/10.3233/AIS-130234

Copyright: IOS Press

http://www.iospress.nl/

Postprint available at: Linköping University Electronic Press

http://urn.kb.se/resolve?urn=urn:nbn:se:liu:diva-102111 


\title{
A personalized exercise trainer for the elderly
}

\author{
Gabriele Bleser ${ }^{\mathrm{a}, *}$, Daniel Steffen ${ }^{\mathrm{a}}$, Markus Weber ${ }^{\mathrm{a}}$, Gustaf Hendeby ${ }^{\mathrm{b}, \mathrm{c}}$, Didier Stricker ${ }^{\mathrm{a}}$, \\ Laetitia Fradet $^{\mathrm{d}}$, Frédéric Marin ${ }^{\mathrm{d}}$, Nathalie Ville ${ }^{\mathrm{e}}$, and Francois Carré ${ }^{\mathrm{e}}$ \\ ${ }^{a}$ German Research Center for Artificial Intelligence, Department Augmented Vision, Kaiserslautern, Germany. \\ \{firstname.lastname\}@dfki.de \\ b Swedish Defence Research Agency (FOI), Department Sensor Informatics, Linköping, Sweden. \\ gustaf.hendeby@foi.se \\ ${ }^{\mathrm{c}}$ Linköping University, Department of Electrical Engineering, Linköping, Sweden. hendeby@isy.liu.se \\ ${ }^{\mathrm{d}}$ Université de Technologie de Compiègne, UMR CNRS 7338 Biomécanique et Bioingénérie, Compiègne, France. \\ \{firstname.lastname\}@utc.fr

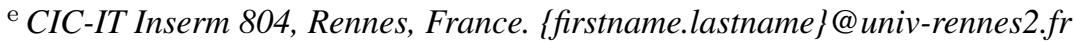

\begin{abstract}
Regular and moderate physical activity practice provides many physiological benefits. It reduces the risk of disease outcomes and is the basis for proper rehabilitation after a severe disease. Aerobic activity and strength exercises are strongly recommended in order to maintain autonomy with ageing. Balanced activity of both types is important, especially to the elderly population. Several methods have been proposed to monitor aerobic activities. However, no appropriate method is available for controlling more complex parameters of strength exercises. Within this context, the present article introduces a personalized, home-based strength exercise trainer designed for the elderly. The system guides a user at home through a personalized exercise program. Using a network of wearable sensors the user's motions are captured. These are evaluated by comparing them to prescribed exercises, taking both exercise load and technique into account. Moreover, the evaluation results are immediately translated into appropriate feedback to the user in order to assist the correct exercise execution. Besides the direct feedback, a major novelty of the system is its generic personalization by means of a supervised teach-in phase, where the program is performed once under supervision of a physical activity specialist. This teach-in phase allows the system to record and learn the correct execution of exercises for the individual user and to provide personalized monitoring. The user-driven design process, the system development and its underlying activity monitoring methodology are described. Moreover, technical evaluation results as well as results concerning the usability of the system for ageing people are presented. The latter has been assessed in a clinical study with thirty participants of 60 years or older, some of them showing usual diseases or functional limitations observed in elderly population.
\end{abstract}

Keywords: physical activity monitoring, elderly, home-based rehabilitation, HCI, wearable sensors, health promotion, strength exercises, personalization, ambient assisted living

\section{Introduction}

Regular and moderate physical activity practice provides many physiological benefits, reduces the risk of disease outcomes, and generates important psychological gains [32]. Aerobic activity, promoting cardiovascular fitness, and strength exercises, promoting musculoskeletal fitness, are strongly recommended. Particularly in frail populations, balanced activity of

\footnotetext{
*Corresponding author. E-mail: gabriele.bleser@dfki.de
}

both types is important to keep functional independence $[14,32]$. It is hence essential to promote the practice of physical activity, especially at home, since it has been proven that adherence to exercise is greater when performed at home than when performed in centers $[1]^{1}$. However, this physical activity has to be supervised in order to improve physical fitness whilst minimizing the risk of injuries due to overuse or in-

\footnotetext{
${ }^{1}$ This has been shown in people older than 50 years showing cardiovascular and respiratory diseases or risk factors
} 
adequate physical activity practices $[5,10]$. For that purpose the supervision of physical activity has to be achieved whilst providing feedback and motivational elements to the users regarding their activity. Such supervision aims to preserve or increase motivation and program adherence.

Within this context, the present article introduces the PAMAP $^{2}$ platform that supervises, motivates and helps the practice of physical activity. This system has been developed within the European Ambient Assisted Living project PAMAP ${ }^{3}$ and has been designed for promoting home-based physical activity adapted to the elderly population. The PAMAP system provides a holistic way of physical activity monitoring by supporting guidance and follow-up of both typical aerobic activities, to promote cardiovascular health, and more complex strength exercises, to improve or maintain strength and balance. While the overall system is briefly introduced, this article focuses on the major novel part of the system dedicated to the supervision of strength exercises, the personalized exercise trainer.

The article is organized the following way: Section 2 provides a short overview of the related work. Then, Section 3 starts with presenting the end-user perspectives of the overall PAMAP system. It describes the effects of aging on mental and physical health and fitness [2] and how these effects should be taken into account when designing the user interface for the elderly. Furthermore, the requirements from health care professional point of view are highlighted. Then, Section 4 briefly outlines the technical realization of the complete PAMAP system, before introducing the strength exercise monitoring use case, which this article focuses on. The next section, Section 5, presents the user interface in relation to the requirements stated in Section 3. The underlying activity monitoring solution is then described in the two following sections: Section 6, detailing the full-body motion capturing based on wearable inertial sensors, and Section 7, describing the novel methodology for personalized exercise evaluation and the parameters of exercise load and technique to be controlled. The results of the system and user evaluation are presented in Section 8, and conclusions are drawn in Section 9.

\footnotetext{
${ }^{2}$ Physical Activity Monitoring for Aging People

${ }^{3}$ Project AAL-2008-1-162, http: / / www . pamap. org
}

\section{Related work}

The supervision of physical activity at home has until now mainly been related to rehabilitation follow-up. Information and Communication Technologies (ICT) have been used for this purpose by providing rehabilitation services at home over telecommunication networks and Internet. This constitutes the recent field of telerehabilitation [25]. However, only a few of these projects have tried to provide a more complete approach of services including monitoring of physical activity, a wellness diary, mentoring sessions, and a web-portal to facilitate personal goal setting and to assess the progress of each patient in the program [31]. Additionally, most often these telerehabilitation projects focus on a specific aspect of physical activity related to a special disease or pathology and do not consider physical activity as a whole [19].

In the field of physical activity monitoring, recently developed methods based on wearable (mostly inertial) sensors have been proposed to monitor aerobic activities [4,13,18,27]. With such methods it is possible to control, whether the F.I.T.T. (Frequency, Intensity, Type, Time) principles of training [5] are well respected by the user. A similar approach has also been integrated into the PAMAP system [22,24]. Some systems and methodologies have also been presented to monitor and supervise home-based motor retraining and coordination exercises especially for stroke patients' rehabilitation, but none addressed more complex strength exercises (see [18] for a thorough review of wearable sensors and systems with application in rehabilitation). Examples include the Philips Stroke Rehabilitation Exerciser [11] and Hocoma's ValedoMotion $^{4}$, which both focus on the monitoring of specific body parts (back, knee, elbow) with few wearable inertial sensors. Current video games include feedback based on wearable motion or external vision sensors in order for users to follow some fitness exercises. While such gaming systems are motivating and can have a positive effect on strength, balance and overall fitness, only few and undocumented parameters are taken into account leading to a lack of proper monitoring and helpful feedback. Moreover, the available systems are not personalizable for users with specific needs and individual limitations and their use in frail populations has led to injuries as reported in a recent survey [28]. Finally, external vision sensors, such as

\footnotetext{
${ }^{4}$ http: //www. hocoma.com/products/valedo/ valedomotion/
} 
Microsoft's Kinect, suffer from the line-of-sight problem and therefore restrict the training panel that can be monitored to exercises, which allow full frontal visibility of the person and which are performed indoors.

Until now, no appropriate method for capturing and monitoring and personalized controlling of the complex parameters related to the load and technique of full-body strength exercises in a home-based environment is available. Therefore, the present article proposes a novel methodology and presents its realization and integration in the aforementioned PAMAP system.

\section{End-user requirements}

The end-user perspective of the PAMAP system can be divided into two parts: the perspective of the elderly population and the perspective of the health care professionals. The clinical partner involved in the PAMAP project, specialized in cardiovascular diseases and physical activity rehabilitation for patients and elderly, has defined the system requirements based on the characteristics of the elderly population and their own expectations from a clinician point of view, thus providing the framework for system design.

For the first part, three main aspects and effects of aging on mental and physical health and fitness [2] have been considered: the cognitive changes with ageing (decreased ability to perceive a high amount of information at the same time, decrease of memory), the physiological changes (decrease of sensory abilities, e.g. vision and hearing, decrease of movement accuracy and coordination) and the ability to deal with recent technology. The cognitive and the physiological changes should be taken into account during the design of the user interface ( $c f$. Section 5) and the design of the wearable sensors ( $c f$. Section 6). For the user interface, the quantity of information presented to the user should be reduced to the most useful and simplest form and should be presented by different sensory means (visual, auditive). The information should also be repeated at each use in order to avoid problems due to a decrease of memory. The interaction with the system as well as the manipulation and the fixation of the wearable sensors should not require any fine motor skills. Finally, the user interface should be integrated into a system familiar to the user in order to limit the required learning of unknown technology. During the system design phase, a few participants were also informally asked to test different solutions and provide feedback for iterative improvements.
The requirements from the health care professionals' point of view concerned the information provided to the user during the physical activity monitoring, the parameters to control in order to ensure a good physical activity practice and to detect risks, and, finally, the parameters to be accessible by health care professionals. The clinical partner specified the protocol for a physical activity session the user has to follow (warmup phase, exercise, cool-down phase), including the duration of each phase and the period of rest to respect between each exercise set. Moreover, he defined the parameters of the aerobic exercises (duration and intensity) and of the strength exercises (number of sets and repetitions, movements in terms of speed, amplitude, smoothness and plane of motion). He also defined the parameters to be controlled in order to avoid risks (acceptable heart rate intervals, feeling scales, postures during strength training) and the tutorials the user should have access to in order to learn about physical activity practice, recommendations, and adverse symptoms. Finally, the clinical partner specified the data to enter into the system (training program, medical data), and the data to receive about the user (medical data, physical activity program realization).

A detailed documentation of the user requirements is given in [29]. These requirements provided the basis for the PAMAP system design and technical realization as detailed in the following sections.

\section{Pamap system overview}

The PAMAP system has been developed according to the user requirements stated in Section 3. The complete platform covers a large spectrum of different home based health activities, from logging the user's daily activity profile (as detailed in e.g. [22]) to coaching specific strength activities. Section 4.1 provides a technical perspective of the overall system. The article then focuses on the strength exercise monitoring use case, which represents a major contribution to the state-of-the-art of physical activity monitoring.

\subsection{Technical system overview}

The overall PAMAP system is modular and flexible: As illustrated in Figure 1, it is composed of four self-contained principal components that communicate over a network. The end user interacts with the data presentation and data management components. The underlying physical activity monitoring function- 


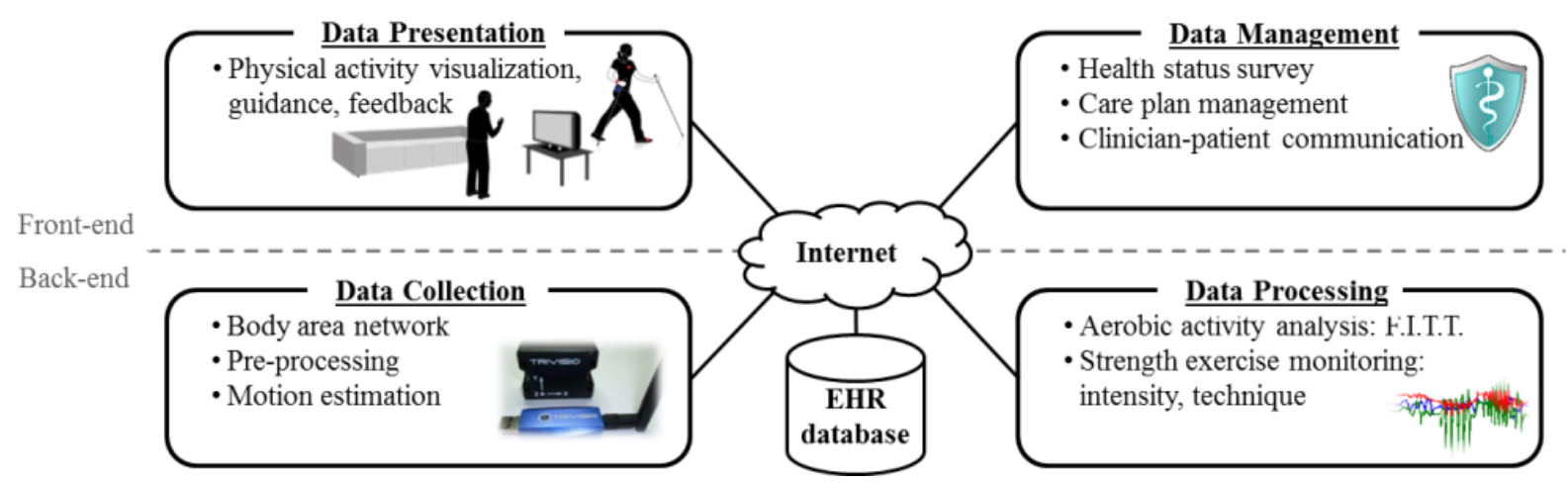

Fig. 1. PAMAP system overview. The upper components represent the part of the system that the user interacts with, the lower components encapsulate the underlying technology for physical activity monitoring and the electronic health record (EHR) database.

ality is encapsulated in the data collection and processing components.

The data presentation component provides visualization, guidance and feedback while exercising. Individual user interfaces have been developed for the different categories of physical activity. Particular effort has been spent in developing the personalized strength exercise trainer. Based on an interactive TV (i-TV), the user is guided through a personal training session consisting of a warm-up phase, a work phase with different strength exercises, and a cool-down phase as specified in Section 3. During training, instant audio and visual feedback is provided on the way the exercises are executed. This feedback is based on the comparison of the performed motion as captured by the data processing component with a previously recorded personal gold standard motion. After each exercise, the user receives feedback on the overall quality of execution. This enables self-monitoring and can motivate users to increase their performance during the next session.

The data management component comprises an electronic health record and a care management application with web and i-TV interfaces. These applications enable management, sharing and reviewing of collected activity data, and facilitate health care professionals to maintain comprehensive medical records of their patients, and in the establishment and follow up of personalized rehabilitation and physical activity plans for them. A more detailed description of this component can be found in [26]. It is not in the focus of the present article.

The data collection component is based on a network of (wireless) wearable sensors and a mobile processing unit for capturing the user's motions and vital signs. The complete set-up combines up to ten minia- ture inertial sensors and complementary sensors, such as a heart rate monitor. However, the system is designed in a modular way, so that the available sensors determine the physical activity parameters that can be monitored [23]. Preprocessing includes correction, filtering and synchronization of the raw sensor data, as well as, the derivation of higher-level information, for instance, the body posture in terms of joint angles. Wearable inertial sensors have been chosen for capturing the user's motions, since these don't suffer from the line-of-sight-problem and therefore allow for a greater action radius and set of exercises to be monitored.

The data processing component analyzes and characterizes the physical activity of the user based on the captured data. Several individual algorithms have been developed to enable sophisticated analysis. As indicated in the introduction, these range from the derivation of general F.I.T.T. parameters to personalized evaluation of strength exercises, the latter being the focus of this publication.

\subsection{Use case: personalized strength exercise trainer}

Fig. 2 illustrates the PAMAP system when being used as a personalized strength exercise trainer. The aim is to guide a user through an individualized training session, to accurately evaluate the performed movements with respect to the parameters specified in Section 3 and, based on this, to provide valuable feedback in real-time ( $c f$. Section 5). In short, the aim is to help the user to perform the training correctly and safely. However, what is correct depends on the user's mobility, possible limitations and physical activity goals. Hence, personalized exercise monitoring, respecting these aspects, is required. 


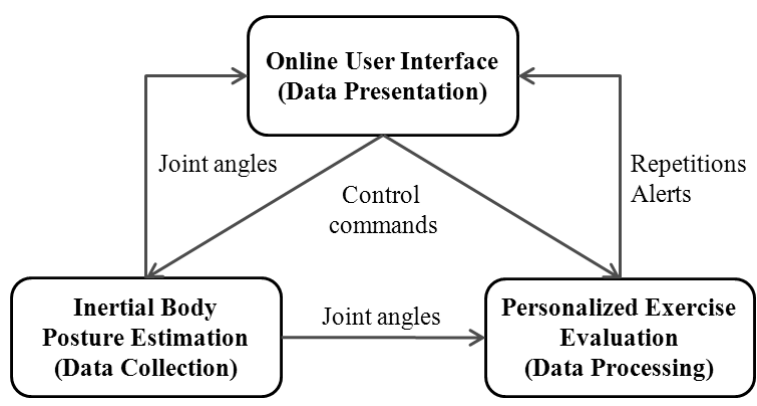

Fig. 2. The different system components and how they interact in the personalized exercise use case. Note that this figure represents an instantiation of the general system illustrated in Figure 1.

The basic idea for achieving this in a generic way is depicted in Figure 3. The proposed solution is to create a personalized reference for evaluation within a first supervised training session. During this session, the proposed system is used in a teach-in mode. While the user is shown how to perform the exercises according to his or her special needs, motion sequences are captured based on the wearable sensor network ( $c f$. Section 6). These are then further processed to derive a personalized model for each exercise ( $c f$. Section 7.1). At home, the system is used in the trainer mode during exercising. In this mode, the performed movements are evaluated by comparing them to the stored reference models ( $c f$. Section 7.2). The evaluation results are then translated into helpful audio and visual feedback for the user ( $c f$. Section 5).

\section{Data presentation}

This section explains the data presentation component, the part of the system that the user interacts with and that presents the information provided by the other system components. The focus is on introducing a user interface for older adults based on the requirements as specified in Section 3. After outlining the overall functionality, the general design concept is introduced and then illustrated with different use cases.

\subsection{Functionality}

The aim of the personalized exercise trainer is to help the user to follow his/her individually tailored training program when exercising alone at home. In particular, this means to guide the user through the different phases of the program including preparation, warm-up, exercise, and cool-down phase; to control the exercise load by suggesting the weights, number of sets and repetitions, and breaks according to the training plan; to function as a virtual memory by counting the number of sets and repetitions; and to control the exercise execution by providing valuable feedback on the way the exercises are performed. The latter are based on the information provided by the data processing component.

\subsection{User interface design principles}

One of the most difficult problems for elderly users to adopt technology is the usage of interfaces that are often not well-suited for them. Especially, the aspect of technology acceptance needs to be addressed. The elderly users should be unaware of the complexity of the underlying software and hardware. The technology should aim at being less noticeable than traditional hardware and better integrated with the lifestyle of the elderly [15]. Moreover, the interface should be developed for elderly people with little or no computer experience. The core philosophy is therefore a minimalist design and simplicity of the user interface.

Currently, the set-up for the elderly makes use of a television. The users are able to enter information interactively via a remote control. Using a television the
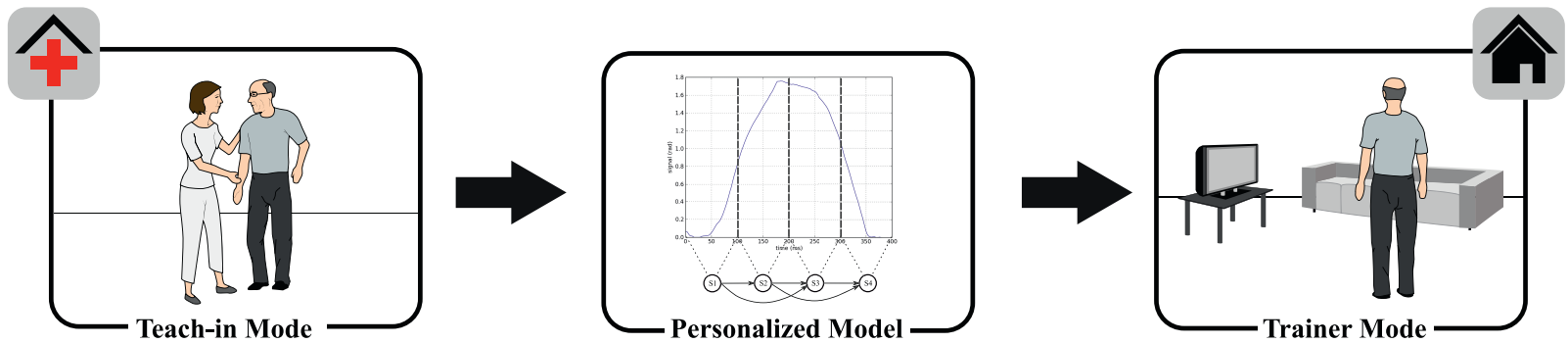

Fig. 3. Overview of the personalized exercise trainer use case. During the guided Teach-in Mode a reference motion is recorded. Based on the captured motion data a Personalized Model is generated. In the Trainer Mode at home the model is used as personal evaluation reference. An individual model is trained for each exercise. 


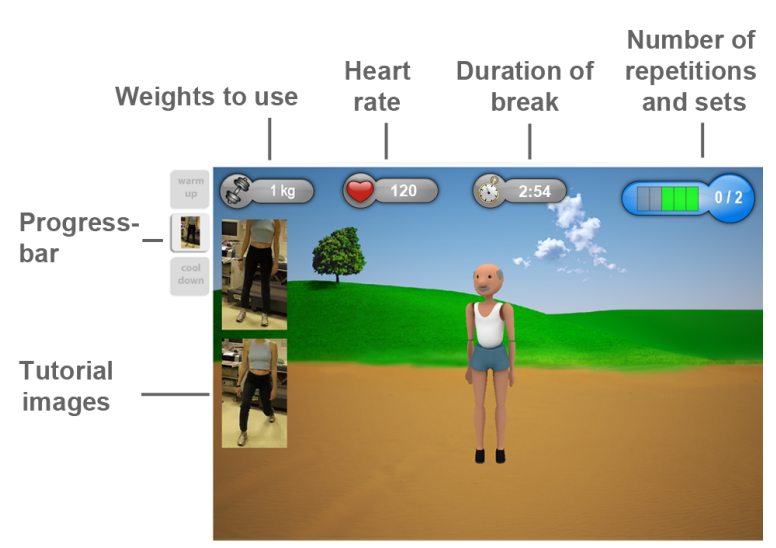

Fig. 4. Overview of the user interface during a strength exercise. Different design elements (progress bar, tutorial images, etc.) help guiding the user through an individual session.

need to learn a new interface is negligible, as the user will utilize an already familiar interface [7]. Furthermore, the user is unaware of any underlying software or hardware details within the system, as the monitor visually and behaviorally represents a television with the remote control still being the primary input device.

Besides the wearable sensor network, the graphical user interface is the main component with which the user gets into contact. Modern interface design is very demanding when it comes to memory and sight, while these abilities decrease with age [35]. This process includes a decline over time in the cognitive and physical functions at different rates relative to each other. Visual changes among aging adults include problems with reading speed, seeing in dim light, reading small print, and locating objects.

In the current system the symbols were designed to be simple and large. A large and clear font is also used in the application and only essential information is displayed on the screen ( $c f$. Fig. 4). To support the presented text information a speech output has been introduced for limited vision users. At any point in time the PAMAP system explains what the user has to do next.

\subsection{User interface design elements}

An overview of the user interface design elements is shown in Fig. 4. These are the main elements to help guiding the user through various types of strength exercises. The user's movements are visualized by means of a virtual avatar. A body-shape representation has been chosen based on a study by Cui et al [3], which shows that both female and male users preferred a body-shape avatar over a stick-figure or mirror-image

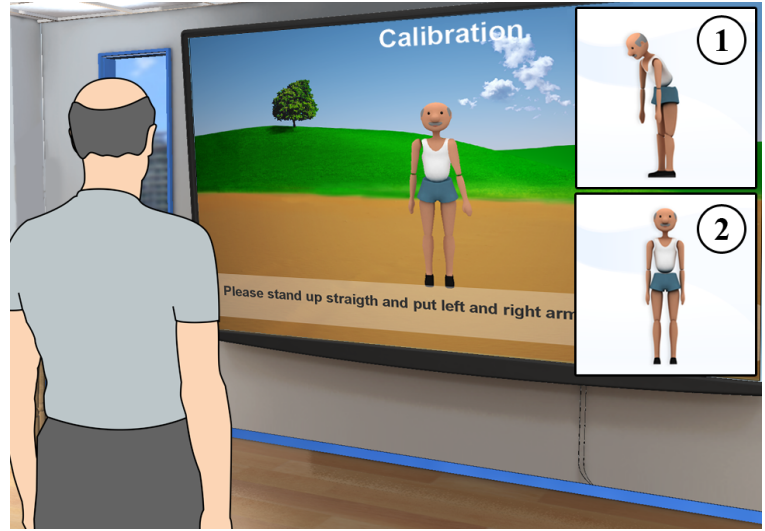

Fig. 5. By means of visual (text and images) and audio instructions the personalized trainer guides the elderly user through the two-step calibration process.

avatar when seeing and sharing their exercise movements. In terms of personalization the user can select a male or female avatar during startup of the PAMAP application.

The progress bar indicates the current phase of the training session including warm-up, cool-down and exercise. When pressing the play button on the remote control during an exercise the user can switch to a tutorial video showing how to perform the exercise correctly and which tools or weights should be used. The remaining elements are dedicated to the current exercise: tutorial images, the weights to use, the duration of a break, and the number of repetitions and sets to perform and already completed support the user while exercising. When wearing a heart rate monitor, the user's heart rate can also be visualized.

The following sections describe two different use cases with respect to the user interface: the calibration procedure and the strength exercise.

\subsubsection{Use case: calibration}

The motion estimation based on inertial sensors requires a simple calibration procedure ( $c f$. Section 6.2) in order to ensure repeatable and accurate motion data. Therefore, special work has been undertaken to ensure a valid calibration process.

Fig. 5 illustrates, how the system leads the user through the two-step procedure. With text, images, and audio instructions, the user is asked to bend over and then to stand upright with arms and legs straight down. When the calibration is completed, the system is ready to visualize the user's movements through his virtual alter ego. 

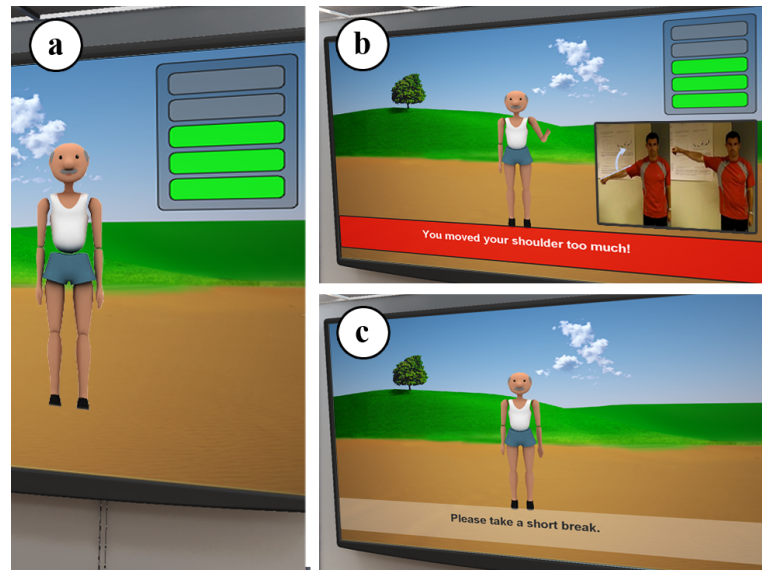

Fig. 6. Interfaces during an exercise: Besides the number of repetitions (a), the system provides feedback in case of wrong execution (b) and protects against overexertion (c).

\subsubsection{Use case: strength exercise}

Fig. 6 shows the user interfaces during a strength exercise such as biceps curls. Here, the user only carried out three of five repetitions ( $c f$. Fig. 6a). During the execution the system serves as virtual memory. It has the knowledge of the type of exercise, the number of repetitions and sets to perform, and the successfully carried out repetitions and sets. Hence, the elderly user can concentrate on correctly executing the exercises.

Furthermore, the system intervenes when an exercise is performed incorrectly. If the user does not respect the exercise load or technique as assessed by the data processing component, a dedicated feedback is provided as textual warning message ( $c f$. Fig. 6b), explanatory image and complementary audio message. The feedback is based on the evaluation parameters that have not been respected (see Section 7.2.2): movement speed, movement amplitude, movement smoothness, joint rotation axes, and posture.

Finally, the system is capable of protecting against overexertion. Regular breaks between the exercise sets are part of the training session ( $c f$. Fig. 6c). Thus, the risk of an overuse injury, which is of specific interest for users with cardiac disease or functional limitation, is minimized. In addition, with the respective equipment, an alarm on the cardiac frequency can be provided.

\section{Data collection and motion estimation}

In order to accurately evaluate the exercises whilst being performed, precise body motion estimation is re- quired in real-time. This section describes how this information is deduced from the data of wireless inertial measurement units (IMUs) attached to the user's body.

\subsection{Inertial measurement units (IMUs)}

The PAMAP system can make use of up to ten IMUs carefully positioned on the user's body to track his or her movements. In principle, the system works with any IMUs providing synchronized measurements of sufficient quality and that have a form factor that allows the user to comfortably wear the sensors. In this case, sensors have been developed in close cooperation with the company Trivisio ${ }^{5}$. The resulting IMUs are wireless $(56 \times 42 \times 17 \mathrm{~mm}, 41 \mathrm{~g})$ and contain a 3axis MEMS accelerometer and gyroscope, and a 3-axis magnetometer, all sampled at $100 \mathrm{~Hz}$.

\subsection{Inertial body posture estimation}

A drawing of the IMUs in relation to the upper body model is depicted in Figure 7. The pose and motion

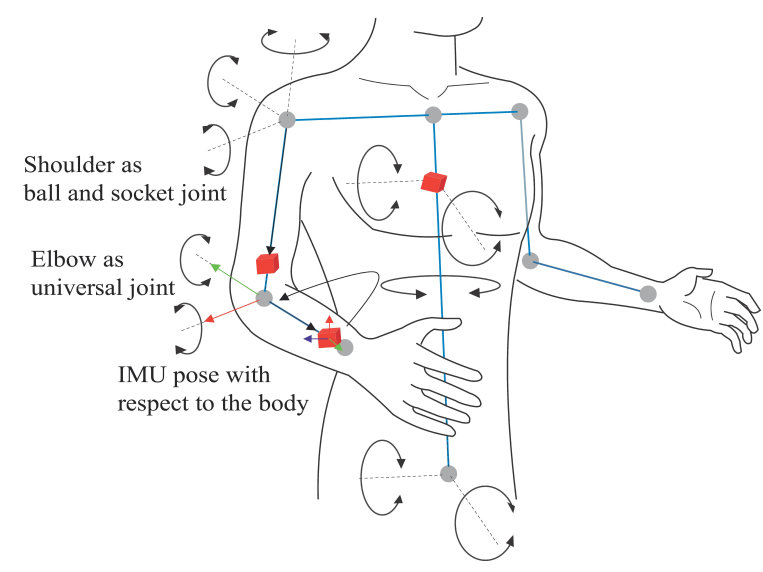

Fig. 7. Functional upper body model with indicated IMU placement (red cubes). The model for the lower body is structured analogously.

of the body are derived from the measured accelerations, angular velocities, and magnetic fields from the IMUs. These measurements are compared to predictions based on a biomechanical body model. The pose kinematics are then determined using model based sensor fusion.

The biomechanical model determines the degrees of freedom (DOF), and based on this, the set of movements that can be captured. In the context of the pro-

5http://www.trivisio.com 


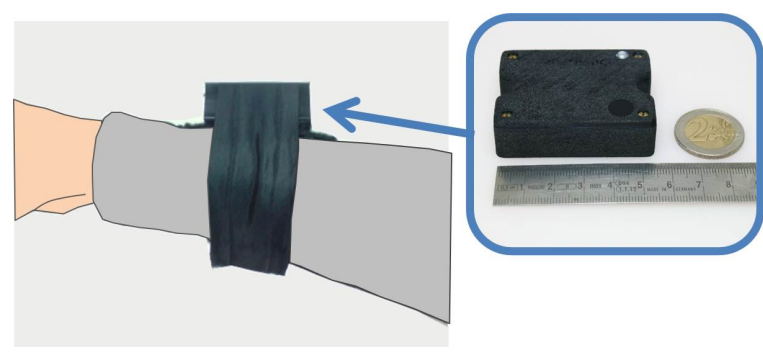

Fig. 8. Schematic of the fixation used on the lower arm and a sensor closeup.

posed system, the model is a compromise between simplicity and accuracy: It is detailed enough to provide accurate data for the exercise evaluation and movement visualization through the avatar. At the same time the model is mathematically tractable and thus enables an efficient real-time estimation. The complete model is a functional model consisting of ten rigid bodies (torso, pelvis, upper arms, forearms, upper legs, and lower legs) connected by anatomically motivated restricted joints. The model is parameterized by the segment lengths, so that it can be personalized for the individual subject.

At least one IMU is required at each segment that should be monitored in order to obtain its complete orientation. To accurately capture the whole body pose, ten sensors are needed. The sensors are placed as indicated in Figure 7. The positions are chosen with respect to several constraints: it should be unobtrusive and limit the skin and muscle motion artifacts. Therefore, IMUs are placed on bones, ligaments, and between muscles. In order to guarantee convenient usage and precise data, an easy, fast and repeatable positioning of the IMUs on the body is required. Moreover, the fixation method should neither allow for too many degrees of freedom nor be too size-dependent. The proposed solution is based on a modified sports suit. At the IMU locations, velcro is fastened in order to enable fixing the sensors to the suit at pre-defined locations, but with some flexibility in positioning. Elastic silicon placed onto the other side of the suit under the IMUs has been added to limit the IMUs' motions. Moreover, separate velcro straps made of elastic fabric and adjustable in size have been developed to cover the IMUs and provide additional stability. Fig. 8 shows a closeup of the final sensor casing and fixation.

To be able to use the IMU measurements to estimate the body posture, the poses of the sensors, more specifically, their orientations relative to the body segments must be calibrated. For this, an easy to perform cali- bration procedure based on IMU measurements taken under known static poses has been developed ( $c f$. Section 5.3.1). The devised procedure requires the user to bend over and then to stand upright with their arms and legs straight down and the thumbs and feet forward. The relative orientations are then deduced from the measured accelerations and magnetic fields.

The estimation of the body posture in terms of joint angles, angular rates and accelerations is carried out by a set of nested extended Kalman filters (EKFs) [9]. The configuration of the limbs (arms, legs) and the trunk segments are each estimated in an individual EKF. The EKFs are then loosely coupled. The measurement equations are based on forward kinematics and the functional model presented above. Given the joint angles from the filter and the model, the body pose is fully determined. The algorithm is described in more detail in [21].

\section{Data processing}

As previously mentioned and depicted in Fig. 3, exercise evaluation comprises a teach-in mode and a trainer mode.

The aim of the teach-in mode is a personalized gold standard that can serve as a reference for one motion cycle. The idea is to learn this model from correctly performed example executions. For this, motion data (according to the user's abilities) is collected during the teach-in mode, where the user is supervised by a physical activity specialist.

In order to provide the functionality of the trainer mode, the aim is to automatically detect the exercise repetitions and to accurately evaluate each repetition based on the respective reference motion.

\subsection{Teach-in mode}

This section presents a fully automated method to construct a reference model from a very short training sequence. Fig. 9 illustrates an example sequence of forty seconds of a biceps curl exercise. The training data in terms of recorded joint angles is assumed to contain a predefined number of exercise repetitions correctly performed by the user. A Hidden Markov model (HMM) is used to represent the reference model. This representation has been chosen for two reasons: Firstly, it naturally takes variations in motion into account by allowing for time-warping and has thus been successfully applied in domains such 


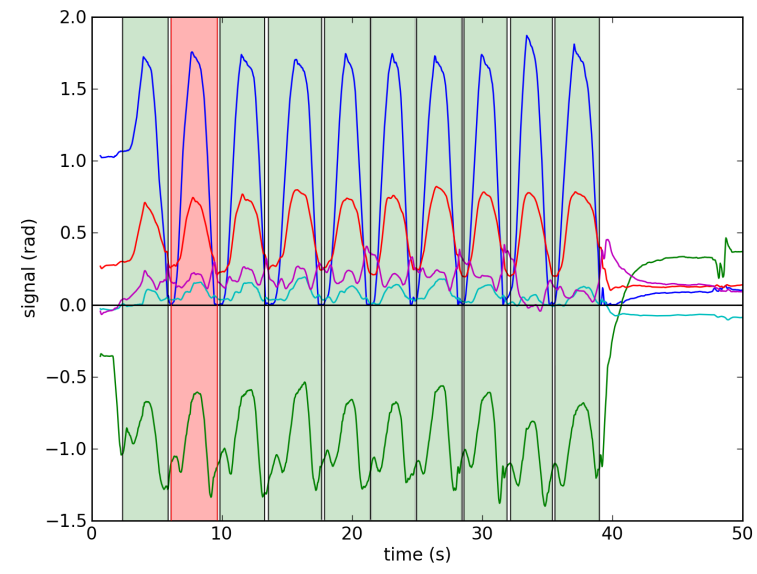

Fig. 9. Training signal for a biceps curl exercise: The five graphs show the measured joint angles in terms of Euler angles of the right arm during a guided training session. The best motif candidate (red) and its occurrences (green) are marked.

as speech [20], gesture [12], or handwriting recognition [8]. Secondly, standard algorithms, such as the Viterbi algorithm [20], can be used for segmentation. The proposed method for model generation consists of two steps: The first step automatically detects candidates for the recurring motion cycles in the training sequence. The second step uses the detected motion cycles to construct the model.

\subsubsection{Candidate detection}

The problem of locating patterns in real-valued, multivariate time series is a known problem and several approaches have been proposed [16,17]. However, all of these methods are based on a predefined window size. Here, the windows size is equivalent to the time needed to execute one exercise repetition. Since the execution time is unknown, the first step is to estimate a suitable window size. Based on the assumption that the exercise repetitions during the teach-in mode are performed consecutively with roughly the same speed, a dominant frequency will be present in the signal. This can be extracted using the combined power spectral density (PSD) [34]. The window size is then initialized as the period of the dominant frequency. Based on this preprocessing step, an extended version of [16] is then parameterized with the window size to detect the reference motion candidates. The best candidate is evaluated and chosen as final result. Fig. 9 illustrates an example of a candidate reference motion cycle and its segmented occurrences in the training signal.

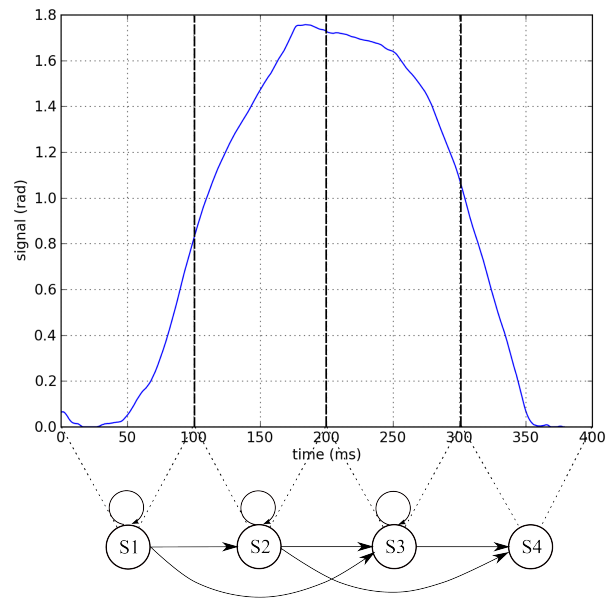

Fig. 10. Personalized Model: HMM for one channel of the signal.

\subsubsection{Model generation}

The observation probabilities of the HMM are modeled as Gaussian mixtures. Here, the different channels (joint angles) are handled separately, so that the system is capable of identifying, in which angle a deviation occurs. The reference motion cycles which have been extracted during the candidate detection are used as training samples for the model. Since traditional parameter estimation methods for HMMs, such as the Baum-Welch algorithm, typically fail when being applied to too few training examples, a simple construction algorithm is used to capture the characteristics of the reference motion. This algorithm builds a HMM with a left-right topology, which is a wide-spread approach to model time-varying sequential data. Selftransitions and skip-transitions are added to allow for a faster and slower execution of the motion. The number of hidden states is chosen in relation to the estimated window size. Accordingly, each subsequence is divided into equal-length adjacent segments, whereas each segment is assigned to a state. Fig. 10 illustrates an example model with four states. For each state a Gaussian mixture is trained using an expectationmaximization algorithm on all elements of the respective training set. Thus, each segment is described by a normal distribution.

\subsection{Trainer mode}

The trainer mode consists of two phases that build upon each other. First, the reference model is used to detect and segment the repetitions while being performed. During this step, deviations from the reference motion are already identified, but not further character- 
ized. Once, a repetition is completed, the second phase consists in accurately evaluating the quality of the performed motion cycle according to the evaluation criteria as introduced in Section 3 and further detailed below.

\subsubsection{Motion cycle detection}

As already stated, the HMMs obtained during the teach-in mode enable the online detection of the represented reference motion within continuous motion data by means of the Viterbi algorithm. In general, the standard Viterbi algorithm computes the most likely path of states given a sequence of observations. Here, the observations are given by the continuous joint angles as streamed by the data collection component. Thus, the algorithm can determine, to which state, respectively frame, of the reference motion the current motion matches. If the probability of the Viterbi algorithm is below a defined threshold, the current observation is considered to be not the correct motion. The motion cycle detection immediately allows for counting exercise repetitions and deducing their duration. Whenever a complete motion cycle has been detected, the detailed evaluation starts.

\subsubsection{Motion cycle evaluation}

According to the requirements stated in Section 3 and the literature on strength training, it is fundamental to check that the load of the exercise as well as its technique are respected in order to ensure effectiveness and safety. The load of the exercise, the muscles that work but also the posture taken during the exercise have then to be evaluated. Translating these constraints into objective data that are implied in the measurements resulted in the following criteria: For movement load, the exercise intensity is quantified by the number of repetitions, the movement speed, the movement amplitude, and the movement smoothness. For the muscles to work, it was chosen to characterize the axes of rotation. Finally, for safety issues, it was also chosen to characterize the posture by evaluating fixed distances or angles that are recommended when performing the movement. This could, for instance, be the distance between the feet during squat exercises, or the angle at the pelvis during push-ups.

The number of repetitions and their duration are given by the motion cycle detection step described above. For the other criteria, an algorithm has been developed, which evaluates each detected motion cycle using the model constructed during the teach-in mode as reference. The different steps of the algorithms are the following. First, different fixed angles and dis-

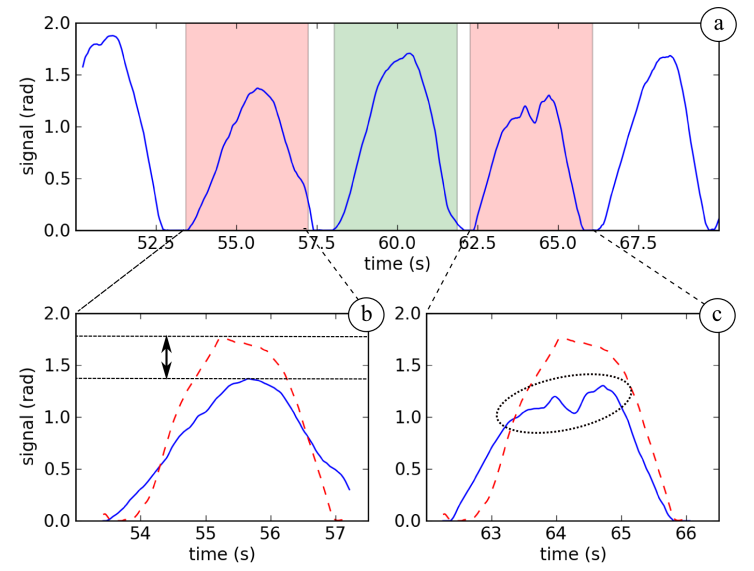

Fig. 11. Training Mode: The personalized model depicted in Fig. 10 is used to evaluate the signal of one joint angle (a). The detected motion cycles are evaluated separately. The cycles overlaid with a red area show a significant deviation from the reference movement (illustrated as dashed red lines), either in amplitude (b) or in the number of extrema (c). The green area indicates a correctly performed cycle.

tances that must be respected during the movement in order to avoid injuries are computed and compared with those obtained during the movement of reference. Afterwards, the principal rotation axis is computed for the current cycle at each joint. The principal rotation axes are then compared to the ones obtained during the movement of reference. Using the same formalism, the rotation amplitudes are also compared. Finally, the number of extrema (i.e. local maxima or minima) in the derivative of the joint trajectory (i.e., its velocity) that has the greatest range of motion during the movement is evaluated and compared in order to determine movement smoothness. The procedure is illustrated in Fig. 11. The movement duration manifested in the velocity, the fixed angles and distances and the rotation amplitudes of the movement to evaluate should not differ by more than a certain threshold from the reference model. The principal rotation axes should not deviate more than a certain threshold from those obtained from the reference. For the smoothness, the same number of extrema has to be found, since any other number of extrema translates a deviation and in particular a parasite movement or a break in the movement realization. If any of these abovementioned criteria are not met, an alert is generated and sent to the user interface, which translates this into explanatory feedback. The motion cycle evaluation concept is summarized in Fig. 12. 


\begin{tabular}{|c|c|c|c|}
\hline Constraints & Parameters & Measures & Thresholds \\
\hline Safety & Posture & $\begin{array}{l}\text { Fixed angles and distances } \\
\text { at/between joints }\end{array}$ & $\pm x \%$ of reference value \\
\hline \multirow[t]{4}{*}{ Load of exercise } & $\begin{array}{l}\text { Number of repeti- } \\
\text { tions }\end{array}$ & Number of cycles & Same as reference \\
\hline & Movement velocity & Movement duration & $\pm x \%$ of reference value \\
\hline & Movement amplitude & $\begin{array}{l}\text { Range of motion from } \\
\text { quaternions at moving joints }\end{array}$ & $\pm x \%$ of reference value \\
\hline & $\begin{array}{l}\text { Movement smooth- } \\
\text { ness }\end{array}$ & $\begin{array}{l}\text { Number of extrema in veloc- } \\
\text { ity of most moving joint }\end{array}$ & Same as reference \\
\hline Muscles to work & Joint rotation axes & $\begin{array}{l}\text { Quaternion axes at moving } \\
\text { joints }\end{array}$ & $\begin{array}{l}\text { angle deviation, } \theta \text {, from ref- } \\
\text { erence axis }\end{array}$ \\
\hline
\end{tabular}

Fig. 12. Motion cycle evaluation concept: The fixed angles and distances are provided by the clinical staff. The other measures are directly deduced from the movement of reference. The values $x$ and $\theta$ are parameters of the algorithm.

\section{System evaluation}

The main focus of this work was on introducing a novel methodology for being able to monitor both the load and technique of full-body strength exercises, and to implement this methodology in a personal trainer user interface, which should help older adults, healthy or with disabilities, to exercise safely and correctly in a home-based environment. Accordingly, this section describes technical evaluation results concerning the feasibility and performance of the proposed monitoring methodology, as well as, quantitative and qualitative results concerning the usability and acceptance of the proposed system in ageing people, assessed within a clinical assay.

\subsection{Technical evaluation}

The goal of this technical study is to assess the performance of the integrated personalized exercise monitoring system, as described in Sections 6 and 7 with respect to capturing, detecting and evaluating motion cycles of previously learnt exercises. For a detailed evaluation of the inertial body motion tracking, the reader is referred to [6].

\subsubsection{Participants}

Seven participants took part in this study: five males and two females. They were mainly employees or students at research institutes or universities, age $27.5 \pm$ 5.30 years and were more or less physically active.

\subsubsection{Materials}

The participants' movements were captured using the inertial motion capture system as described in Section 6. Here, one sensor was placed on each segment of the upper-extremities, namely, on each forearm, on each upper arm, on the upper-torso, and on the pelvis as indicated on Fig. 7.

\subsubsection{Procedure}

The participants performed eight strength training exercises typical in cardiac patients' rehabilitation. Only upper-extremity exercises were considered, since they generally exhibit a greater variation in the amplitude and joints to work and since they are generally less familiar to beginners. The number of series and the posture to respect (e.g. the back should be straight) were those described by the exercise program. For each exercise, the participants performed a first series under the supervision of a physical activity teacher involved in the study. This series was used to generate the reference model. During the other series, the participants could freely choose to respect the number of repetitions and the recommendations provided by the rehabilitation program or to modify their posture, the movement range of motion, the speed, the rhythm, and/or the number of repetitions. The motion cycle detection and evaluation algorithms were then applied to each series of exercise. The tolerances for detecting incorrect movements were initially set to 20 percent for the movement speed, amplitude and posture. For the rotation axes, an angle deviation of $20^{\circ}$ was accepted. A physical activity teacher annotated the movements for the ground truth.

\subsubsection{Results and discussion}

Fig. 13 presents the confusion matrix of the exercise evaluation obtained with the above mentioned tolerances. For the number of repetitions and the velocity of each movement for each exercise the algorithm shows good correspondence with the human annotations. For the other parameters, satisfactory results could be obtained when the tolerance between the movement of 


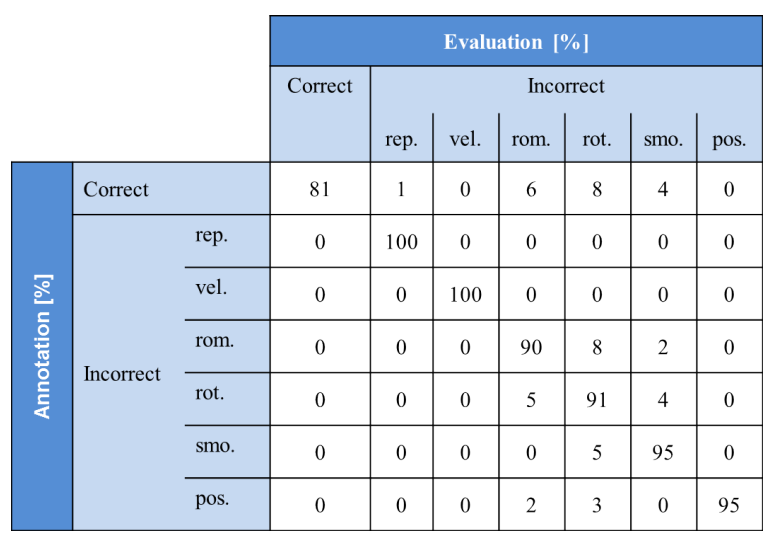

Fig. 13. Confusion matrix of the exercise evaluation in percentage when tolerating a deviation of $20 \%$ and $20^{\circ}$ from the movement of reference. The ground truth annotations were provided by a physical activity teacher involved in the study. The abbreviations are according to the evaluation parameters in Section 7.2.2: number of repetitions (rep.), movement speed (vel.), movement amplitude (rom.), movement smoothness (smo.), axes of rotation (rot.) and posture (pos.). Here, each line adds up to $100 \%$. The entries on the diagonal show the $\%$ of correct classifications in each category, while the off-diagonal entries show the $\%$ of misclassifications, given the above mentioned parameter settings. As an example for the first row: $81 \%$ of the executions annotated as correctly executed were also classified as correctly executed by the algorithm, while $9 \%$ of the executions annotated as correctly executed were misclassified by the algorithm as either not adhering the velocity (1\%), or not adhering the range of motion $(6 \%)$ and so on.

reference and that actually evaluated was increased. The algorithm was indeed less tolerant to deviations from the movement of reference than the physical activity teacher. With a threshold of 30 percent for the movement speed, amplitude and posture and a tolerance of $30^{\circ}$ for the joint rotation axes, only the movements that looked incorrect to the examiner were also detected as being incorrect by the algorithm. While these results prove the feasibility of the proposed algorithms, they also indicate the necessity to further investigate the thresholds chosen for detecting and classifying incorrect motions. The thresholds might be a function of the subjects and the movements to evaluate. A too strict evaluation should be avoided, since it might discourage or disturb the participant more than being of help.

\subsection{Clinical study}

This section outlines the clinical assay of the final PAMAP system in terms of design, conduction and results. Here, the focus was on the overall acceptance and usability of the system for the target group of ageing people.

\subsubsection{Participants}

The trials population was chosen to be representative of the ageing general population (from the point of view of usual diseases or functional limitations observed in elderly population) for whom a regular physical activity practice was possible and highly recommended for maintaining health and preventing disease complications. Hence, a mixture of healthy participants and people showing current chronic diseases was focused. Altogether, 30 French participants (recruited in sports associations, fitness centers, a cardiovascular prevention department and a functional rehabilitation department) completed the study. Ages ranged from 60 to 85 (mean $69.17 \pm 6.77$ years). Among these, ten participants, three women and seven men, aged $67.2 \pm 5.1$ years were healthy, ten participants, three women and seven men, aged $68.7 \pm 6.5$ years suffered from cardiovascular disease, and ten participants, six women and four men, aged $71.6 \pm 8.3$ years had functional limitations. All participants had normal or corrected-to-normal vision and 19 participants have already used a game console like Nintendo's Wii or Sony's PS3 with motion tracking.

\subsubsection{Materials}

The participants' movements were captured using the inertial motion capture system as described in Section 6. Here, ten IMUs were used, one on each segment of the upper and lower extremities. Five sizes of sports suits allowed choosing the best size for each individual, both for males and females ( $c f$. Fig. 15, left).

\subsubsection{Procedure}

The study was organized in supervised sessions over nine days. Each day three to four of the overall 30 participants came to the medicine department for testing the personal exercise trainer over approximately two hours (from arrival to departure).

Each participant had to perform eight strength exercises. Among these eight exercises, three were performed by all participants, whereas five were specific to each group (healthy participants, cardiovascular disease group, functionally disabled group). In addition, the group of functionally disabled participants was divided into functionally disabled with respect to upper or lower body. These four individualized training sessions had been prepared by the clinical partner involved in the PAMAP project and could be selected via the PAMAP user interface ( $c f$. Fig. 14).

The protocol consisted of three phases: (1) a teachin phase, where all exercises were performed once under human coach supervision; (2) a familiarization 


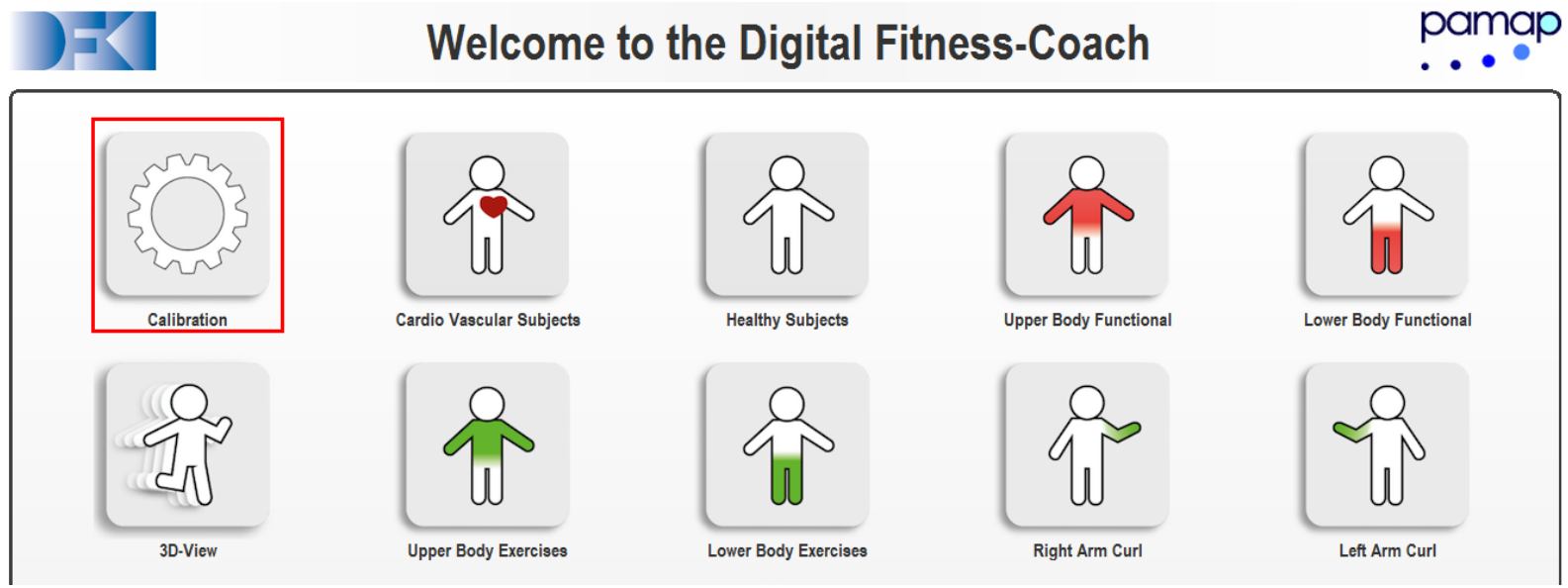

Fig. 14. Part of the welcome screen of the strength exercise trainer system indicating the different functionalities and exercise programs. The user interface is available in three languages: French, English and German.

procedure, where the participant could interact with the PAMAP user interface through the remote control, watch a tutorial video and try out the different functionalities ( $c f$. Fig. 15, middle); (3) the measurement phase, where the participant was guided through the calibration and then through his/her individual training session ( $c f$. Fig. 15, right).

This last phase was again divided into two subphases: (3a) a semi-automatic mode, where five exercise were performed without any feedback related to the quality of the movement; (3b) a full automatic mode, where three exercises were fully guided by the PAMAP system based on the exercise monitoring algorithms presented in Section 7.
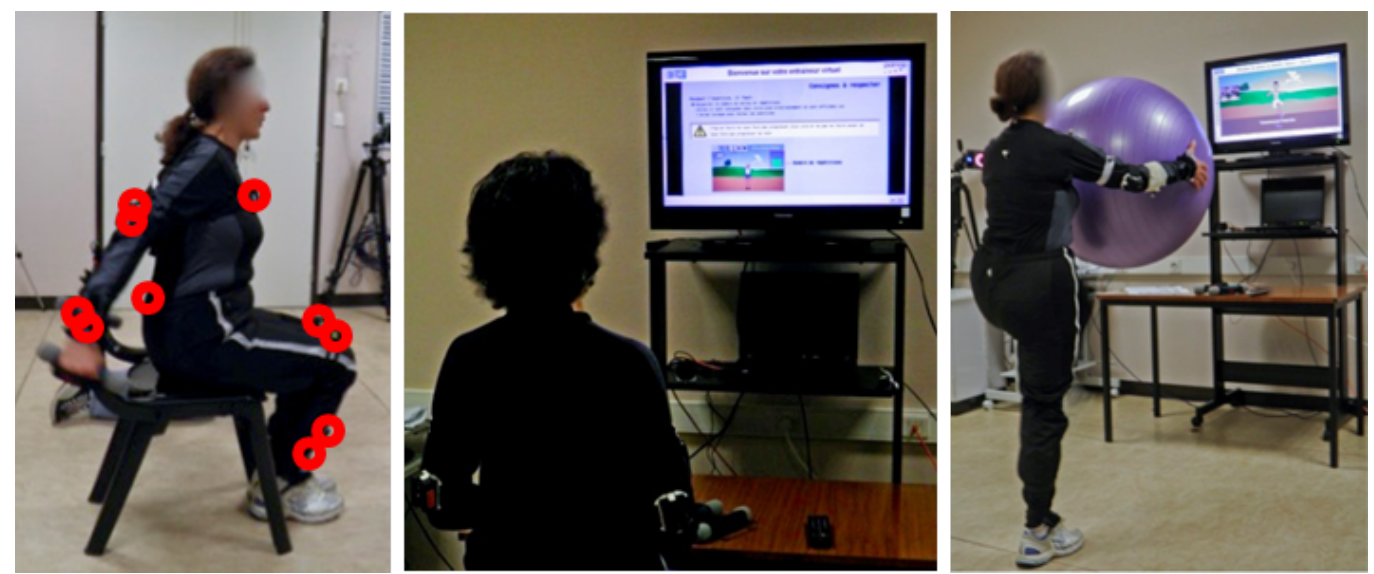

Fig. 15. Clinical study. Left: sports suit with attached IMUs (indicated by red circles); middle: a participant watching the tutorial video on the TV screen during the familiarization procedure; right: a participant performing her exercise program while being guided by the proposed strength exercise trainer system.
The semi-automatic phase was used to evaluate the ease with which the participant interacted with the PAMAP system and performed the exercise program. The automatic phase was used to test the developed movement evaluation algorithm (see [33] for detailed results) and to evaluate the acceptance of such extended functionalities among the participants. The human coach and two system engineers were present all along the session without intervening after the teach-in mode. At the end of the training session, the participant filled in a questionnaire related to the PAMAP system. The questionnaire contained questions on the participants' general impression of the interface, its usability and its suitability to the task, provided on a 7-item Likert scale. Here, a value of one represents a strong 
disagreement and a value of seven a strong agreement with the given statement. Moreover, the participant was invited to describe his or her thoughts about the guided exercise session, with the advantages and limits of the system. The preparation and conduction of the clinical assay is described in more detail in [30].

\subsubsection{Results and Discussion}

The results of the user study including questionnaires, observations and interviews are summarized in the following.

Before the experimental procedure started, many participants showed some apprehension, fearing that they would not manage the new technology. However, after a short demonstration and the familiarization phase including the video tutorial, all participants were able to use the PAMAP system without help. They found the system easy to interact with and the instructions they were given easy to understand. This fact also reflects in the questionnaire regarding ease-of-learning (mean $6.1 \pm 0.759$ ), ease-of-use (mean $6.2 \pm 0.961$ ) and clarity of the user interface (mean $6.5 \pm 0.731$ ).

Concerning the PAMAP system idea in general, the participants found it useful to train at home following the prescription of a professional sports coach or physician. Only one participant answered that it was far from likely to use the PAMAP system in everyday life. In general, the participants were in favor of using such technology on a regular basis (mean $5.1 \pm$ 1.78). They also appreciated the possibility for health care professionals to be informed about their sessions and the quality of their movements. This functionality is provided by the data management component of the PAMAP system ( $c f$. Section 4.1).

Concerning the data presentation during exercise execution, the evaluation of the questionnaires showed that all information was presented clearly, legibly, and comprehensibly regarding vision (mean $6.63 \pm 0.556$ ) and audio (mean $6.47 \pm 0.776$ ). However, the size of the tutorial pictures explaining the exercises should be increased, since it was observed that some participants approached the screen when looking at them. The human coach also observed that, during some exercises, the participants tended to hold their breath or did not breathe in agreement with the movement. Hence, videos could also be used to explain the correct breathing or additional sensors could be added to supervise the breathing and provide real-time feedback.

In general, despite initial doubts, all participants enjoyed the technology and were very interested in seeing their avatar move on the TV screen. They consid- ered this as a nice aid for correcting their posture accurately and in real-time in order to avoid injuries. In particular, the automatic mode, with its counting of the repetitions, switching automatically from one exercise to the next and providing feedback, when a movement was incorrectly performed, was fully appreciated and helped the participants to correct their movements. Moreover, all participants were pleasantly surprised by the encouragements given by the system through motivating feedback after each exercise and at the end of the session.

Some valuable suggestions for enhancing the PAMAP system were also contributed by both the participants and the involved health care professionals. The videos and pictures explaining the exercises could show the movements from different perspectives, e.g. a frontal and lateral perspective, in order to improve the understanding.

Some participants wished further possibilities for personalization, e.g. choosing or setting up their own avatar (in addition to choosing between a male or female avatar) and configuring the visualization on the TV screen (mirrored vs. non-mirrored perspective).

Different levels of instructions (e.g. beginner, advanced, expert) were also proposed in order to provide an interface adapted to the physical activity practice and familiarization of the user with the system.

\section{Conclusion and future work}

This article has presented the PAMAP system, a powerful platform for promoting home-based physical activity adapted to the elderly population. While providing a short overview of the overall system and its functionalities, the article focuses on the special use case of the system as a personalized strength exercise trainer. This digital trainer helps the user at home to follow his or her exercise program, to perform the exercises correctly, and to stay involved in the practice of physical activity. The user interface has been specifically designed for older adults with little or no computer experience. In order to provide instant feedback during exercising, a novel methodology for capturing and monitoring full-body strength exercises by means of a wearable sensor network has been proposed. The method evaluates the exercise load as well as the technique whilst taking into account the individual characteristics and goals of the user. This is achieved by means of a supervised teach-in mode, which enables the system to learn personalized gold standard mo- 
tions. At the same time, this technique reduces the dependency of the system on a fixed selection of exercises, since arbitrary motion sequences can be defined as exercises.

The feasibility and the usability of the system have been evaluated in technical studies and a clinical assay showing promising results in both the potential of the novel technology and the acceptance among the elderly population. Indeed, the clinical assay showed a real interest and appreciation of the proposed technology among the elderly.

Based on these positive results, the PAMAP system is currently further developed, taking into account the feedback and experiences from the evaluation phases, as well as, the latest developments in terms of available miniaturized sensory devices. Another part of future work consists in the enhancement of the motion cycle segmentation and evaluation algorithms including automatic data-driven parameter selection in order to ease the usability for health care professionals and achieve good detection and classification results. A further interesting extension of the motion cycle evaluation would be a continuous adaptation of the reference models to the performance and progress of the user, thus avoiding the need for a repeated teachin mode, which is currently necessary. However, for safety issues, regular medical checkups and reference model teach-in under supervision of a physician would still be necessary in the case of patients. Finally, besides the envisaged technical improvements of both the monitoring methodology and the user interface, an important aspect of future work will be to investigate ways to maintain and increase long-term motivation of the elderly to stay involved in physical activity practice. Here, we will focus on the design of digital games for elderly to encourage usage of the developed platform and by this improve their level of physical activity.

\section{Acknowledgements}

This work has been performed within the project PAMAP funded under the AAL Joint Programme (AAL-2008-1). The authors would like to thank the project partners and the EU and national funding authorities for the financial support. For more information, please visit the website http: / / www . pamap. org.

\section{References}

[1] N. Ashworth, K. Chad, E. Harrison, B. Reeder, and S. Marshall. Home versus center based physical activity programs in older adults. Cochrane Database of Systematic Reviews, 25(1), 2005.

[2] K. Berger. The developing person: Through the life span. Worth Publishers, 2008.

[3] J. Cui, Y. Aghajan, J. Lacroix, A. van Halteren, and H. Aghajan. Exercising at home: Real-time interaction and experience sharing using avatars. Entertainment Computing, pages 63-73, 2009.

[4] S. de Vries, F. Garre, L. Engbers, V. Hildebrandt, and S. van Buuren. Evaluation of neural networks to identify types of activity using accelerometers. Medicine and Science in Sports and Exercise, 2010.

[5] F. W. Dick. Sports training principles. A. \& C. Black, 1997.

[6] L. Fradet, A. Reiss, G. Bleser, I. Lamprinos, N. Ville, and O. Machui. PAMAP - System Evaluation Report. Available at http://www.pamap.org/publications.html (public deliverables), January 2010.

[7] T. Hewett, R. Baecker, S. Card, T. Carey, J. Gasen, M. Mantei, G. Perlman, G. Strong, and W. Verplank. ACM SIGCHI curricula for human-computer interaction. Technical report, New York, NY, USA, 1992.

[8] J. Hu, M. Brown, and W. Turin. HMM based online handwriting recognition. IEEE Transactions on Pattern Analysis and Machine Intelligence, 18(10):1039 -1045, October 1996.

[9] A. H. Jazwinski. Stochastic Processes and Filtering Theory, volume 64 of Mathematics in Science and Engineering. Academic Press, Inc, 1970.

[10] D. Kirkendall. Exercise prescription for the healthy adult. Primary Care, 11(1):23-31, 1984.

[11] G. Lanfermann, J. te Vrugt, A. Timmermans, E. Bongers, N. Lambert, and V. van Acht. Philips stroke rehabilitation exerciser. In International Conference on Telehealth/Assistive Technologies, Anaheim, CA, USA, 2008.

[12] H. Lee and J. Kim. An HMM-based threshold model approach for gesture recognition. IEEE Transactions on Pattern Analysis and Machine Intelligence, 21(10):961 -973, October 1999.

[13] M. Mathie, A. Coster, N. Lovell, and B. Celler. Accelerometry: providing an integrated, practical method for long-term, ambulatory monitoring of human movement. Physiological Measurement, 25(2), 2004.

[14] R. Mazzeo and H. Tanaka. Exercise prescription for the elderly: current recommendations. Sports Medicine, 31:809818, 2001.

[15] B. Meyer, D. Bouwhuis, S. Czaja, W. Rogers, M. Hufschmidt, and J. Fozard. Senior CHI: how can we make technology "elder-friendly?". In CHI '99 extended abstracts on Human factors in computing systems, pages 81-82, New York, NY, USA, 1999.

[16] D. Minnen, C. Isbell, I. Essa, and T. Starner. Discovering multivariate motifs using subsequence density estimation and greedy mixture learning. In National Conference on Artificial intelligence (AAAI), volume 1, pages 615-620, Vancouver, Canada, 2007.

[17] D. Minnen, T. Starner, I. Essa, and C. Isbell. Discovering characteristic actions from on-body sensor data. In IEEE International Symposium on Wearable Computers, pages 11 -18, October 2006 
[18] S. Patel, H. Park, P. Bonato, L. Chan, and M. Rodgers. A review of wearable sensors and systems with application in rehabilitation. Journal of Neuroengineering and Rehabilitation, 9(21), 2012.

[19] B. Pogorelc and M. Gams. Home-based health monitoring of the elderly through gait recognition. Journal of Ambient Intelligence and Smart Environments, 4(5):415-428, 2012.

[20] L. Rabiner and B. Juang. An introduction to hidden markov models. IEEE ASSP Magazine, 3(1):4 -16, January 1986.

[21] A. Reiss, G. Hendeby, G. Bleser, and D. Stricker. Activity recognition using biomechanical model based pose estimation. In European Conference on Smart Sensing and Context (EuroSSC), Passau, Germany, 2010.

[22] A. Reiss, I. Lamprinos, and D. Stricker. An integrated mobile system for long-term aerobic activity monitoring and support in daily life. In International Symposium on Advances in Ubiquitous Computing and Networking (AUCN), Liverpool, UK, 2012.

[23] A. Reiss and D. Stricker. Introducing a modular activity monitoring system. In International Conference of the IEEE Engineering in Medicine and Biology Society $(E M B C)$, Boston, MA, USA, September 2011

[24] A. Reiss and D. Stricker. Aerobic activity monitoring: Towards a long-term approach. International Journal Universal Access in the Information Society (UAIS), 2013.

[25] M. Rogante, M. Grigioni, D. Cordella, and C. Giacomozzi. Ten years of telerehabilitation: A literature overview of technologies and clinical applications. NeuroRehabilitation, 27, 2010.

[26] D. Steffen and I. Lamprinos. Physical activity monitoring for the aging population. In IADIS e-Health, Lisbon, Portugal, July 2012.

[27] E. M. Tapia, S. S. Intille, W. Haskell, K. Larson, J. Wright, A. King, and R. Friedman. Real-time recognition of physical activities and their intensities using wireless accelerometers and a heart rate monitor. In IEEE International Symposium on Wearable Computers, pages 1-4, 2007.

[28] M. Taylor, D. McCormick, R. Impson, T. Shawis, and M. Griffin. Activity promoting gaming systems in exercise and rehabilitation. Journal of Rehabilitation Research and Development, 48:1171-1186, 2011.

[29] N. Ville, G. Bleser, L. Fradet, and I. Lamprinos. PAMAP - Revised User Requirements Report. Available at http: / / www . pamap.org/publications.html (public deliverables), January 2011.

[30] N. Ville, L. Fradet, G. Bleser, I. Lamprinos, and A. Reiss. PAMAP - Report on Clinical Trials. Available at http:// www . pamap.org/publications . html (public deliverables), June 2012.

[31] D. Walters, A. Sarela, A. Fairfull, K. Neighbour, C. Cowen, B. Stephens, T. Sellwood, B. Sellwood, M. Steer, M. Aust, R. Francis, C. K. Lee, S. Hoffman, G. Brealey, and M. Karunanithi. A mobile phone-based care model for outpatient cardiac rehabilitation: the care assessment platform (CAP). BMC Cardiovascular Disorders, 10, 2010.

[32] D. Warburton, C. Nicol, and S. Bredin. Health benefits of physical activity: the evidence. Canadian Medical Association Journal, 174(6):801-809, 2006.

[33] M. Weber, M. Liwicki, G. Bleser, and D. Stricker. Unsupervised motion pattern learning for motion segmentation. In International Conference on Pattern Recognition (ICPR), Tsukuba Science City, Japan, 2012.

[34] P. Welch. The use of fast Fourier transform for the estimation of power spectra: a method based on time averaging over short, modified periodograms. IEEE Transactions on Audio and Electroacoustics, 15(2):70-73, 1967.

[35] M. Zajicek. Special interface requirements for older adults. In Workshop on Universal Accessibility of Ubiquitous Computing: Providing for the Elderly, Alcacer do Sal, Portugal, 2001. 